\title{
Investigation of Thermal Stability of Non-Newtonian Melt Flows
}

\author{
Chamil Abeykoon \\ Faculty of Science and Engineering, University of Manchester \\ Oxford Road, M13 9PL, Manchester, UK \\ Chamil.abeykoon@manchester.ac.uk
}

Polymers are one of the major classes of raw materials available for many aspects of production globally. Extrusion serves as a fundamental method for processing polymeric materials. Being poor thermal conductors these materials are difficult to heat and cool and also exhibit high viscosity in their molten state. Hence, heat transfer and rheology are major aspects of polymer processing applications. Also, polymer melts usually exhibit a non-Newtonian behaviour and hence quite complicated to model. This work aims to observe the thermal behaviour/quality of a number of industrially common polymeric materials under a wide range of processing conditions using a capillary die with a specifically designed thermocouple mesh sensor. The melt temperature is considered as the main parameter of determining the thermal quality of the melt. Materials were processed under different screw speeds, set temperature conditions with three different screw geometries. Additionally, a number of other parameters such as the melt pressure, mass throughput, and energy consumption were also observed. The results showed that the uniformity of temperature across the melt flow varied considerably with increase in screw rotational speed whilst it was also shown to be dependent upon process settings, screw geometry and material properties. Moreover, it appears that the effects of the material, machine and process settings on the quantity and quality of the process output are heavily coupled with each other and this may cause the process to be difficult to predict and variable in nature. In terms of the flow behaviour, regardless the processing conditions used, the highest melt temperature was observed always in the middle of the melt flow. At lower processing speeds, the lowest melt temperature was close to the die wall where this position shifted a few millimetres away from the die at higher speeds by forming low temperature shoulder region (making the melt flow highly thermally inhomogeneous). Furthermore, the importance of the use of the appropriate thermal monitoring techniques was also highlighted in determining the actual melt thermal quality during polymer processing applications.

Keywords: Melt temperature, Polymer processing, Process monitoring Non-Newtonian flow, Melt pressure, Fullydeveloped flow, Thermal stability. 